University of Wollongong

Research Online

Faculty of Engineering and Information

Faculty of Engineering and Information

Sciences - Papers: Part A

Sciences

$1-1-2014$

\title{
Electro-mechanical modelling and identification of electroactive polymer actuators as smart robotic manipulators
}

\author{
Rahim Mutlu \\ University of Wollongong, rm991@uowmail.edu.au \\ Gursel Alici \\ University of Wollongong, gursel@uow.edu.au \\ Xingcan Xiang \\ University of Wollongong, xx985@uowmail.edu.au \\ Weihua Li \\ University of Wollongong, weihuali@uow.edu.au
}

Follow this and additional works at: https://ro.uow.edu.au/eispapers

Part of the Engineering Commons, and the Science and Technology Studies Commons

Research Online is the open access institutional repository for the University of Wollongong. For further information contact the UOW Library: research-pubs@uow.edu.au 


\title{
Electro-mechanical modelling and identification of electroactive polymer actuators as smart robotic manipulators
}

\begin{abstract}
Electroactive polymer (EAP) actuators, also known as artificial muscles, have remarkable properties such as low energy consumption, low weight, low actuation foot-print, compliance and bio-compatibility. Several methodologies have been proposed to model and analyse their quasi-static bending behaviour with negligible attention paid to their dynamic behaviour. We, therefore, report on an enhanced methodology to model their highly non-linear bending behaviour by treating them as smart and soft robotic manipulators. The methodology consists of an inverse kinematic model and a dynamic model. The proposed methodology accurately estimates the EAP actuator's whole shape deflection using optimization-based inverse kinematic solutions integrated with an electro-mechanical dynamic model. The experimental and numerical results are presented to show the effectiveness of the soft robotic manipulator model in estimating the highly non-linear bending behaviour of the polypyrrole electroactive polymer (PPy-EAP) actuators. The proposed methodology can easily be extended to other bending type actuators and active smart manipulators. 2014 Elsevier Ltd. All rights reserved.
\end{abstract}

\section{Keywords}

electroactive, robotic, polymer, manipulators, actuators, electro, mechanical, modelling, identification, smart

\section{Disciplines}

Engineering | Science and Technology Studies

\section{Publication Details}

Mutlu, R., Alici, G., Xiang, X. \& Li, W. (2014). Electro-mechanical modelling and identification of electroactive polymer actuators as smart robotic manipulators. Mechatronics, 24 (3), 241-251. 


\title{
Electro-mechanical modelling and identification of electroactive polymer actuators as smart robotic manipulators
}

\author{
Rahim Mutlu ${ }^{a}$, Gursel Alici ${ }^{\mathrm{a}, \mathrm{b}, *}$, Xingcan Xiang ${ }^{\mathrm{a}}$, Weihua $\mathrm{Li}^{\mathrm{a}}$ \\ ${ }^{a}$ School of Mechanical, Materials and Mechatronic Engineering, University of Wollongong, NSW 2522, Australia \\ ${ }^{\mathrm{b}}$ ARC Centre of Excellence for Electromaterials Science, University of Wollongong, NSW 2522, Australia
}

\section{A R T I C L E I N F O}

\section{Article history:}

Received 15 November 2012

Accepted 3 February 2014

Available online 24 February 2014

\section{Keywords:}

Electroactive polymer actuator

Soft robotic actuator

Electro-mechanical modelling

Parameter identification

Nonlinear constrained optimization

\begin{abstract}
A B S T R A C T
Electroactive polymer (EAP) actuators, also known as artificial muscles, have remarkable properties such as low energy consumption, low weight, low actuation foot-print, compliance and bio-compatibility. Several methodologies have been proposed to model and analyse their quasi-static bending behaviour with negligible attention paid to their dynamic behaviour. We, therefore, report on an enhanced methodology to model their highly non-linear bending behaviour by treating them as smart and soft robotic manipulators. The methodology consists of an inverse kinematic model and a dynamic model. The proposed methodology accurately estimates the EAP actuator's whole shape deflection using optimization-based inverse kinematic solutions integrated with an electro-mechanical dynamic model. The experimental and numerical results are presented to show the effectiveness of the soft robotic manipulator model in estimating the highly non-linear bending behaviour of the polypyrrole electroactive polymer (PPy-EAP) actuators. The proposed methodology can easily be extended to other bending type actuators and active smart manipulators.
\end{abstract}

(c) 2014 Elsevier Ltd. All rights reserved.

\section{Introduction}

Over the last decade, smart materials such as electroactive polymers (EAPs) have drawn significant attention due to their interesting properties offering the possibility of new actuation and sensing schemes. These properties include low energy consumption, low weight, low actuation foot-print, compliance, noiseless operation, bio-compatibility and the ability to work in both air and aqueous environments. EAPs have a significant potential for building more efficient robotic applications, sensing or actuating devices or a combination of both. A number of potential devices have been articulated by EAPs: microrobotic gripping systems, energy converters, swimming devices, crawling robots, micromanipulators, stiffness regulators, motion converter mechanisms and many more [1-9] -thanks to their natural muscle-like working principles and their remarkable properties. EAPs, as soft actuators, are especially suitable for biologically-inspired robotics where it is necessary to mimic certain characteristics of natural muscles. For instance, these smart actuators were successfully demonstrated in a swimming robotic fish as artificial muscles powering the caudal fin

\footnotetext{
* Corresponding author at: School of Mechanical, Materials and Mechatronic Engineering, University of Wollongong, NSW 2522, Australia. Tel.: +61 42214115; fax: +61 242215474 .

E-mail address: gursel@uow.edu.au (G. Alici).
}

$[4,5]$. EAPs have been used as actuators in this paper and their modelling and characterisation process have been explained by employing a soft robotic manipulator modelling approach.

Since EAPs were proposed as actuators, several methodologies have been presented in the literature in order to analyse and model the EAP actuators' behaviours based on their chemical, electrical and mechanical properties. In most of these studies, the EAP actuators were treated as a cantilever beam to which an electrical stimulus was applied at its fixed end and its output is measured, at its free end. Pei and Inganas [10] used Timoshenko's classical bending beam theory to estimate the bending displacement of the bi-layer polymer actuators. When the EAP actuator is stimulated, this stimulation produces some strain and therefore, a bending along the actuator. The radius of the curvature formed by the EAP actuator is then measured and used to calculate the magnitude of the strain. This approach was adopted by Benslimane et al. [11], Madden [12] and Alici et al. [13] for tri-layer EAP actuators, however this approach is based on the assumptions of a small strain and constant modulus of elasticity. Alici [14] also applied the classical beam theory, taking non-linear effects into account to estimate the non-linear bending displacements of the PPy-EAP actuators. While these studies focus on the quasi-static bending behaviour of the EAP actuators, they do not consider the dynamic effects. Those studies which rely on quasi-static modelling of an EAP actuator as a 
cantilever beam assume that the EAP actuator's elastic properties are constant. EAP actuators, however, are materials which exhibit change in their properties due to a number of reasons: geometric parameters, substrate synthesis conditions, the type of electrolyte used, the molarity of electrolyte used etc. Therefore, an accurate modelling of the EAP actuators is crucial if they are to be employed in advanced applications such as medical devices, microrobots and artificial organs/muscles. As far as the EAP actuators are concerned, there is still a lack of a reasonably accurate dynamic model which not only estimates the tip position but also predicts their whole bending behaviour as a function of time. One can find several methods to model a system dynamically, but in general, two major methods are commonly used: (i) identifying a transfer function based on the system's input/output behaviour or (ii) analysing the dynamics of the system by a mathematical model using the Newton-Euler method, the Lagrangian or the Hamilton formulations based on the system's physics [15]. While the first method is more suitable for modelling single input single output (SISO) systems, the latter is followed in this study on modelling the EAP actuators as a soft robotic manipulator with a hyper degrees-offreedom. It must be noted that we are not proposing to construct a soft serial robotic manipulator made of EAPs, but to model the EAP actuator as a hyper-redundant soft robotic manipulator using this analogy.

Following the configuration estimation of the EAP actuators based on the inverse kinematic model, the electro-mechanical modelling and the dynamic parameter estimation of the EAP actuator are reported. In our previous papers [16,17], the inverse kinematic shape estimation process was explained in detail in which the EAP actuator's bending curvatures were estimated by solving the EAP actuator's hyper-redundant inverse kinematic model employing an optimization-based method (we introduced and referred to that optimization-based inverse kinematic solution as AngleOPT: angle optimization). We experimentally validated the proposed electromechanical model which can be employed for (i) identifying the stiffness and damping parameters of an EAP actuator's soft robotic model and (ii) controlling the EAP actuator's whole shape deflection under an electrical stimulus without requiring any position and/or deflection feedback information. We establish the dynamic model and identify its parameters experimentally so that the kinematic and dynamic models can be employed to control the output displacement of the EAP actuators using a feedforward control method which does not require external feedback data. Accurate kinematic and dynamic models are needed to estimate the actuator configuration for a given voltage input so that its positioning ability can be improved without using external sensors. It is not practical to use an internal or external sensor to measure the actuator displacement for feedback control. The ideal solution is to have accurate dynamic models and invert these models to control the displacement output.

The main contributions of this paper are developing an explicit electro-mechanical model for an EAP actuator based on the soft robotic manipulator approach and estimating the whole shape behaviour of the EAP actuator dynamically, rather than estimating only its tip position for a given electrical input dynamically or in quasi-static form, as in the previous studies mentioned above. This study also contributes to understanding the behaviour of the EAP actuator's variable dynamic properties (i.e. variable elasticity) by introducing whole actuator modelling and identifying these variable dynamic parameters. It is not straightforward to analyse and model the kinematic and dynamic behaviours of the cantilevered-type EAP actuators used in this paper as their operation principle based on the electrical, chemical and mechanical parameters is not yet fully understood but the electro-mechanical model developed in this paper contributes a greater understanding of these smart and soft actuators' condition-dependent dynamic properties.

\section{Fabrication and operation principles of multi-layer EAP actuators}

In this study, pyrrole monomers are used to fabricate the trilayer laminated EAP actuator's active polymer layers by following a number of steps. First, both sides of a non-conductive porous layer (i.e. polyvinylidene fluoride, PVDF) were sputter coated with gold to prepare a conductive $(<20 \Omega)$ surface for polymerisation. We used lithium triflouromethanesulfonimide (Li.TFSI) as the electrolytic ions. The PVDF layer acts as an electrochemical cell separator and also stores $\mathrm{Li}^{+} \mathrm{TFSI}^{-}$. The commercially available PVDF layer, which is $110 \mu \mathrm{m}$ in thickness, is used as received. A pyrrole monomer $(0.1 \mathrm{M})$ containing polymer growth solution, $\mathrm{Li}^{+} \mathrm{TFSI}^{-}(0.1 \mathrm{M})$ and $1 \%$ water in propylene carbonate $(\mathrm{PC})$ was prepared for the polymerisation process. Then the gold-coated PVDF was placed in the solution. The polypyrrole (PPy) layers were galvanostatically grown from the growth solution at a current density of $0.1 \mathrm{~mA} \mathrm{~cm}^{-2}$ for about $12 \mathrm{~h}$ on the gold-coated PVDF. This polymerisation process provides $\sim 30 \mu \mathrm{m}$ thickness of a PPy layer on each side of the gold-coated PVDF. The laminated PPy-based EAP actuator will be called the PPy-EAP actuator throughout the paper. The PPy-EAP actuator's laminated (tri-layer) configuration and operation principle are depicted in Fig. 1.

The PPy-EAP actuator's operation principle is based on the energy conversion from an electrochemical process to a mechanical output. An electrical input applied to the PPy layers stimulates counter-ions to move in and out of the PPy layers. When the positively charged polymer layer is oxidised, the negatively charged polymer layer is reduced. The TFSI ${ }^{-}$anions move from the electrolyte into the positively charged PPy layer and an opposite reaction happens in the other PPy layer in order to neutralize the charge in the PPy layers. This ion migration causes a volume expansion in the positively charged PPy layer and a volume contraction in the other PPy layer. This electro-chemo-mechanical process therefore generates a mechanical bending in the PPy-EAP actuator, as illustrated in Fig. 1.

\section{Kinematic analysis of EAP actuators as soft robotic manipulators}

Bending behaviours of the EAP actuators can be analysed using the classical beam theories as they have a cantilevered topology, however the classical beam theories assume that material properties such as elasticity modulus are constant. The modulus of

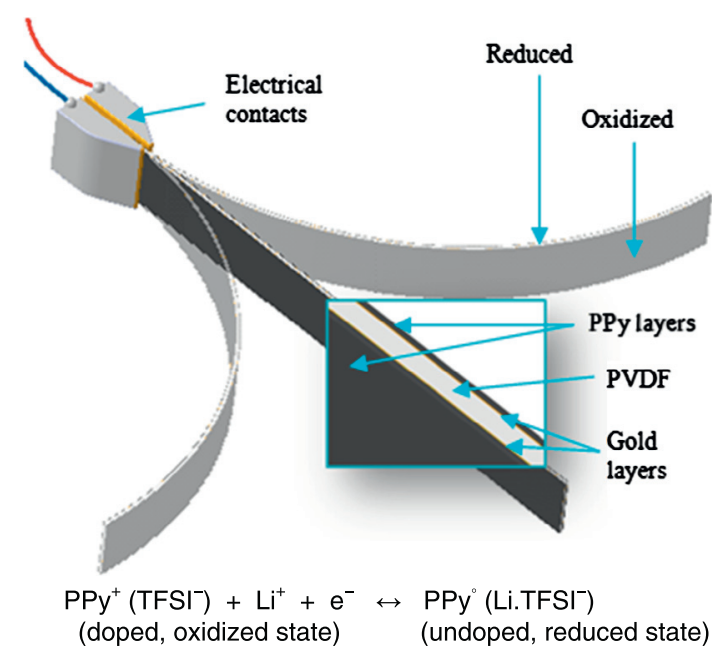

Fig. 1. Structure and the operation principles of the laminated PPy-EAP actuator. 
elasticity of the EAP actuators considered in this study depend on the thickness, width and length of the active polymer layers and the separator, the type of salt, the ionic concentration of the salt, the solvent, and the magnitude and frequency of the potential difference applied. Further, the classical beam theories assume that the beam deflection or the displacement output is below $15 \%$ of the actuator beam (i.e. actuator) length. As has been previously reported, the cantilevered EAP actuators can generate tip deflections as high as $50 \%$ of their length $[18,19]$. Because of this, a different methodology must be followed, taking active material properties into account rather than using a constant property assumption, and small actuator output. The electro-mechanical modelling approach we followed in this study will accurately estimate the EAP actuator's highly non-linear bending behaviour and its dynamic parameters. We employ the soft robotic manipulator approach to model and analyse the bending behaviour of the EAP actuators. In our previous papers $[16,17,20]$, we have demonstrated that the soft robotic manipulator approach can accurately estimate the whole shape bending of the PPy-EAP actuators.

The soft robotic manipulator approach assumes that a backbone curve passes through the geometric centres of the discretised cross-sections of the manipulator or the active structure. This approach calculates the soft robot's kinematic configurations in sequence by calculating the curvature change of this backbone curve. Adapting the soft robotic manipulator approach has been reported before for a snake-like robot [21], an octopus arm [22] and an elephant trunk robot $[23,24]$.

Kinematic analysis of a soft robotic manipulator can be accomplished by employing several methods such as mode shape calculation and optimisation methods. Mode shape method is limited to the case in which movements of the soft robotic manipulator are predetermined for certain modes which should be matched by the backbone curvature of the soft robotic manipulator [21,25-27]. These mode shapes are then incorporated into the forward kinematic model. If the mode shapes which are the kinematic configurations of the soft robotic manipulator are well determined, the mode shape approach can be an effective kinematic analysis method. However, determining these mode shapes can be very problematic depending on the manipulator's configurability and physical constraints. Alternatively, optimisation-based approaches can be employed to calculate the soft robotic actuator/manipulator's backbone from its inverse kinematic model. In this case, the soft robotic actuator is the EAP actuator. Optimisation-based approaches do not require mathematical manipulations, provided that appropriate joint constraints are imposed on the inverse kinematic solutions. We propose to use an optimisation-based approach for which we model the actuator as a hyper-redundant manipulator with serially connected rigid links. The higher the number of the links, the more likely is the configuration correspondence between the real actuator and the hyper-redundant model. With this in mind, we construct an inverse kinematic model of the PPy-EAP actuator and then solve this inverse kinematic model by employing a non-linear constraint optimization method which we call the AngleOPT to estimate all configurations of the actuator throughout its movement under an electrical input. As the thrust of this study is on electro-mechanical modelling of the PPy-EAP actuators, the inverse kinematic model of the PPy-EAP actuator is only briefly presented here. The details of the inverse kinematic model can be found in $[16,17]$.

The backbone curve of the PPy-EAP actuator can be defined with respect to the fixed end of the actuator as shown in Fig. 2

$\boldsymbol{r}(\sigma, t)=\left[r_{x}(\sigma, t) r_{y}(\sigma, t) r_{z}(\sigma, t)\right]^{T}$

where $\boldsymbol{r}(\sigma, t):[0, L] \rightarrow \mathbb{R}^{3}$ assigns a position vector in the Euclidean space to each link (discretised section) parameter $\sigma \in[0, L]$ in time $t$
[28]. $L$ is the overall length of the backbone curve.The position vectors along each axis of a discretised cross-section are described as

$r_{x}(\sigma, t)=\{l \cos \theta \sin \varphi\}_{\sigma}+r_{x}(\sigma-1, t)$

$r_{y}(\sigma, t)=\{l \sin \theta \sin \varphi\}_{\sigma}+r_{y}(\sigma-1, t)$

$r_{z}(\sigma, t)=\{l \sin \varphi\}_{\sigma}+r_{z}(\sigma-1, t)$

where $l$ is the length of each discretised section. Each position vector is described relative to the previous Frenet frame. The whole configuration of the EAP actuator can be obtained by integrating its backbone curve over the discretised sections (differential increments, $d \sigma$ if the EAP actuator is modelled as a continuum system) from its fixed end to the free end. For the tip coordinates $[X, Y, Z]^{T}$ of the PPy-EAP actuator, the objective function to be minimized is given by;

$$
\min _{\theta}\left(f=\sum_{i=1}^{n}\left[\begin{array}{l}
r_{x}\left(\sigma_{i}, t\right) \\
r_{y}\left(\sigma_{i}, t\right) \\
r_{z}\left(\sigma_{i}, t\right)
\end{array}\right]^{-}\left[\begin{array}{c}
X \\
Y \\
Z
\end{array}\right]_{i}\right)
$$

where $r_{x}\left(\sigma_{i}, t\right), r_{y}\left(\sigma_{i}, t\right)$ and $r_{z}\left(\sigma_{i}, t\right)$ are the position coordinates of each section of the EAP actuator, $t$ is time, and $n$ is the number of links. It must be noted that the PPy-EAP actuators used in this study operate in the horizontal plane where the kinematic model reduces to 2-dimensional space.

\section{Dynamics of EAP actuators as soft robotic manipulators}

Dynamic analysis of the EAP actuator is carried out in two steps: (i) obtaining the EAP actuator's equations of motion and (ii) identifying the dynamic model parameters such as stiffness and damping for each joint in the soft robotic manipulator. The dynamic model with the identified parameters is validated in Section 5.

\subsection{Dynamic model}

The dynamic equations are obtained by employing the discretised Lagrangian formulations [29,30]. The kinetic energy of the $i$-th discretised section on the backbone curve can be expressed as

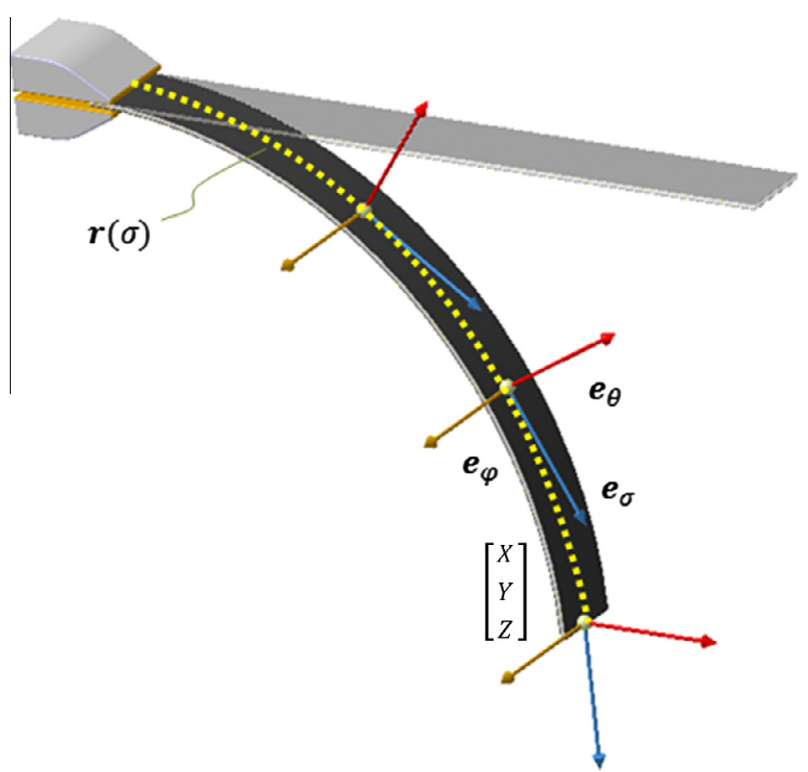

Fig. 2. Configuration of a EAP actuator is defined by its backbone curve, $r(\sigma)$. 


$$
\begin{aligned}
K_{i}(\theta, \varphi, \varepsilon, t)= & \frac{1}{2}\left\{m_{i}\left[\left(\frac{\partial x_{i}}{\partial t}\right)^{2}+\left(\frac{\partial y_{i}}{\partial t}\right)^{2}+\left(\frac{\partial z_{i}}{\partial t}\right)^{2}\right]\right. \\
& \left.+J_{i \theta \varphi \varepsilon}\left[\left(\frac{\partial \theta_{i}}{\partial t}\right)^{2}+\left(\frac{\partial \varphi_{i}}{\partial t}\right)^{2}+\left(\frac{\partial \varepsilon_{i}}{\partial t}\right)^{2}\right]\right\}
\end{aligned}
$$

where $m_{i}$ is the mass and $J_{i \theta \varphi_{\varepsilon}}$ is the inertia tensor of the discretised section measured from the centre of mass of the section which is described as

$J_{i \theta \varphi \varepsilon}=\left[\begin{array}{ccc}I_{x x} & I_{x y} & I_{x z} \\ I_{y x} & I_{y y} & I_{y z} \\ I_{z x} & I_{z y} & I_{z z}\end{array}\right]$

The total kinetic energy is given by

$\mathcal{K}(t) \triangleq \sum_{1}^{n} K_{i}(\theta, \varphi, \varepsilon, t)$

where $n$ is the number of joints or discretised sections along the PPy-EAP actuator's dynamic model. The potential energy of the actuator consists of two components: gravitational potential energy and elastic potential energy. The gravitational potential energy of each discretised section is given by

$P_{g i}(\theta, \varphi, t)=m_{i} g z_{i}(\theta, \varphi, t)$

where $g$ is the gravitational acceleration, $z_{i}(\theta, \varphi, t)$ is the vertical distance between the centre of mass of the $i$-th discretised section and the base plane. The total gravitational potential energy is obtained as

$\mathcal{P}_{g}(t) \triangleq \sum_{1}^{n} P_{g i}(\theta, \varphi, t)$

where $n$ is the number of the discretised sections along the PPy-EAP actuator's dynamic model. The elastic potential energy of the $i$-th discretised section is given by

$$
\begin{aligned}
P_{b i}(\theta, \varphi, t)= & \frac{1}{2}\left[k_{\theta i}\left(\theta_{i}-\theta_{i}-1\right)^{2}+k_{\varphi i}\left(\varphi_{i}-\varphi_{i}-1\right)^{2}\right. \\
& \left.+k_{\varepsilon i}\left(\varepsilon_{i}-\varepsilon_{i-1}\right)^{2}\right]
\end{aligned}
$$

where $k_{\theta i}, k_{\varphi i}$ and $k_{\varepsilon i}$ are the stiffness constants for $\theta, \varphi$ and $\varepsilon$ rotational generalized coordinates, respectively, which represent the elastic bending behaviour of the $i$-th discretised section in three dimensional space. The total elastic potential energy is given by

$\mathcal{P}_{b}(t) \triangleq \sum_{1}^{n} P_{b i}(\theta, \varphi, t)$

where $P_{b i}(\theta, \varphi, t)$ is the elastic energy of the $i$-th discretised section on the EAP actuator model.The Lagrangian function of dynamic equations is given in the following form [30]

$\mathcal{L}(t)=\mathcal{K}(t)-\mathcal{P}(t)$

where $\mathcal{K}(t)$ is the total kinetic energy and $\mathcal{P}(t)$ is the total potential energy of the EAP actuator. Using the Lagrangian function, the generalised motion equations are obtained from

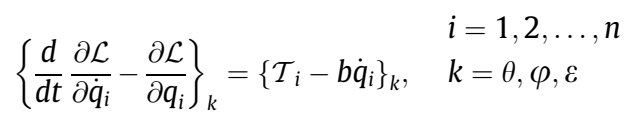

where $q_{i}$ represents generalized coordinates at each joint which are $\theta, \varphi$ and $\varepsilon . \mathcal{T}_{i}$ is the torque (internal bending moment) at $i$-th joint. Also, $i$ and $k$ indicate the joint number from the base frame and the generalized coordinate at the $i$-th joint, respectively. $b$ indicates the damping coefficient in the rotational generalized coordinates which consists of three components: $b_{\theta} b_{\varphi}$ and $b_{\varepsilon}$.

Bending type EAP actuators receive their operation ability from the volume change in their active polymer layers. The stress field induced by the applied electrical stimulus is responsible for the strain field (volume change) in the active polymer layers. The stress field in the EAP actuators can be described as a mechanical load or pressure as a function of the applied electrical stimulus $[7,8]$. This mechanical load as a function of time and input voltage can be obtained by using the blocking force of the EAP actuator [14], as follows

$p_{\text {load }}(t, V)=-\left(\frac{8}{3}\right) *\left(\frac{F_{\text {blocking }}(t, V)}{L}\right)$

where $L$ indicates the overall length of the EAP actuator. The negative sign indicates that the mechanical load is in the opposite direction from the measured blocking force. Using the free body diagram for each discretised section of the EAP actuator, the equivalent bending moment applied at each joint is calculated as

$M_{i}=\sum_{i=1}^{n} p_{\text {load }} \frac{i^{2} l}{2}$

$\mathcal{T}_{i}=M_{i}-M_{i-1}$

where $p$ is given by Eq. (15), $l$ is the length of a discretised section of the EAP actuator. By employing Eq. (14), the equations of motion can be written in a matrix-vector form

$\mathcal{T}(t)=D(q(t)) \dot{q}(t)+H(q(t), \dot{q}(t))+C(q(t))$.

It must be noted that the spatial dynamic model of the EAP actuator is represented by rigid discretised sections (links) and spherical joints which have 3-rotational-DoF, $\theta, \varphi$ and $\varepsilon$. Therefore, the EAP actuator's dynamic model consists of $n \times 3$ nonlinear equations, where $n$ is the number of the discretised sections. The EAP actuator is like a cantilevered beam operating in a 2D plane. This assumption reduces the motion equations from $n \times 3$ to $n \times 1$ equations. Also no gravitational force acts on the EAP actuator, since the EAP actuator operates in the horizontal plane.

\subsection{Dynamic parameter identification}

The elastic behaviour of EAP actuators has been studied using classical strain-stress approaches [31]. The constant elasticity modulus of the EAP actuator assumption was also used in previous studies. The EAP actuators are active smart materials which have properties dependent on several factors including electrical input; ion diffusion; the geometry, porosity and conductivity of the active layers; electrolyte conductivity and molarity. It must be noted that a condition-dependent elasticity modulus denotes conditiondependent joint stiffness values. A similar modelling approach can be found in pseudo-rigid body models for flexural beams in compliant mechanisms [32]. Pseudo-rigid body models can be suitable for passive beams with a constant elasticity modulus; however, for active materials like the EAP actuators, there is a lack of data and methodologies to identify the condition-dependent elasticity modulus due to their complex multi-physics operation principle, which is based on a series of electro-chemo-mechanical processes.

In this paper, we use a direct dynamic parameter identification methodology to identify the joint stiffness and damping parameters of the EAP actuators [29,33,34]. We employ a non-linear least square estimation method to identify the joint stiffness and damping parameters.

Numerical estimation methods are generally sensitive to input data (experimental joint trajectories). The smoother the joint 

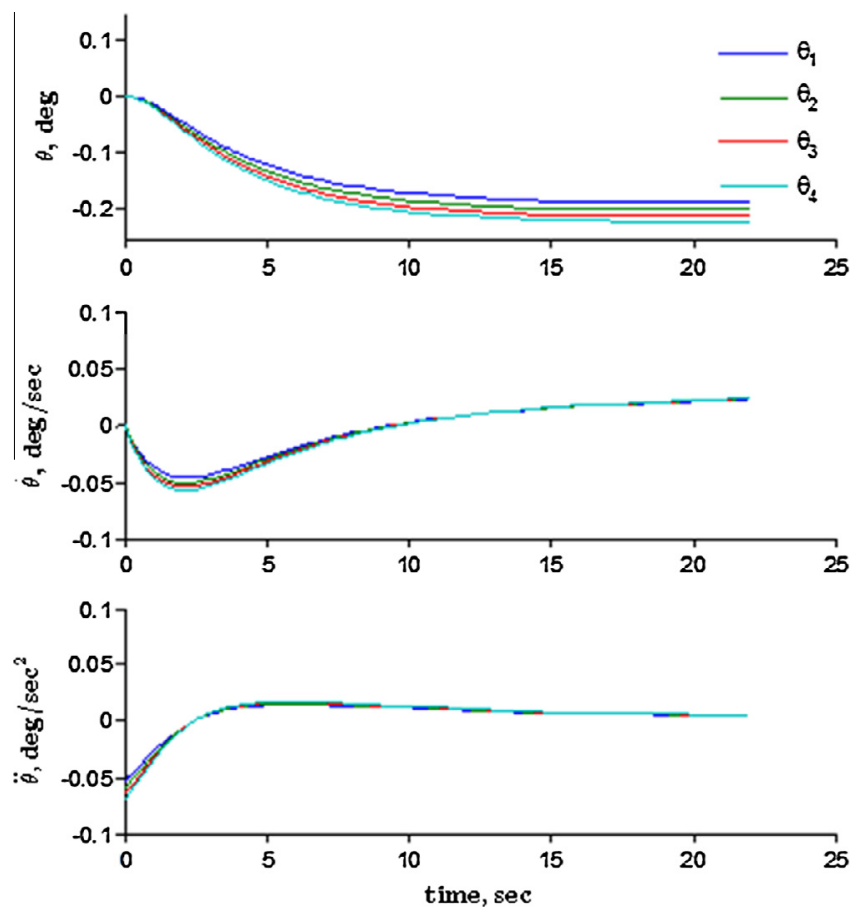

Fig. 3. 4-DoF model joint space trajectories.

trajectories of the EAP actuator's soft robotic manipulator model, the more accurate the dynamic parameter identification becomes. Even though the AngleOPT optimization-based inverse kinematic solutions of the EAP actuator's kinematic model are quite reliable for accurate configuration estimation, special attention is necessary to its boundary/constraint(s) in order to obtain smooth inverse kinematic solutions, which are the joint trajectories to be used in the direct dynamic parameter identification process.

For more accurate parameter identification, we propose to use $s$-curve type harmonic sine and cosine functions fitting to the AngleOPT inverse kinematic solutions,

$$
\begin{aligned}
& \theta_{k}(t)=\sum_{k=1}^{D o F} \sum_{i=1}^{N} A_{i} \cos \left(\frac{t_{i}}{a_{i}}\right) \\
& \dot{\theta}_{k}(t)=\sum_{k=1}^{D o F} \sum_{i=1}^{N} \frac{-A_{i}}{a_{i}} \sin \left(\frac{t_{i}}{a_{i}}\right) \\
& \ddot{\theta}_{k}(t)=\sum_{k=1}^{D o F} \sum_{i=1}^{N} \frac{-A_{i}}{a_{i}^{2}} \cos \left(\frac{t_{i}}{a_{i}}\right)
\end{aligned}
$$

where $A_{i}$ is amplitude and $\frac{1}{a_{i}}$ is the frequency of the harmonic functions, $t_{i}$ indicates time increment, and DoF indicates the number of degrees of freedom. An example of this type of joint trajectory generation for a 4-DoF soft robotic manipulator is demonstrated in Fig. 3. The joint trajectories for the $16-\mathrm{DoF}$ soft hyper-redundant model of the PPy-EAP actuator are obtained using the same procedure. The inverse kinematic solutions with $\Delta t=1 \mathrm{~s}$ increments are shown in the same plots.

Using direct dynamic parameter identification, the EAP actuator's dynamic model parameters, which are joint stiffness and damping values, are estimated using the following non-linear least-square formulation;

$\vartheta=\arg \min _{\vartheta} \sum_{t=1}^{N} \sum_{k=1}^{D o F} \frac{\left(\mathcal{T}_{k}(t)-\mathbf{W}_{\mathbf{k}}\left[\mathbf{q}_{k}(t), \dot{\mathbf{q}}_{k}(t), \dot{\mathbf{q}}_{k}(t), \vartheta\left(k_{k}, b_{k}\right)\right]\right)^{2}}{\sigma^{2}}$

where $\mathbf{W}_{\mathbf{k}}$ is the $k$-th element of the right side of Eq. (18), $\sigma$ is the standard deviation and $\vartheta$ represents unknown parameters (joint stiffness and damping values). This optimization problem can be solved using either Newton interior-reflective or Levenberg-Marquardt iterative search methods. Both algorithms can handle large scale non-linear problems.

\section{Experimental validation}

In this section, we present the implementation and validation of the kinematic and dynamic models of the EAP actuators. To this aim, we used PPy-based EAP actuators with the dimensions of $20 \times 3 \times 0.17 \mathrm{~mm}$, length, width and thickness, respectively. The actuators are modelled as a soft robotic actuator (the approach is

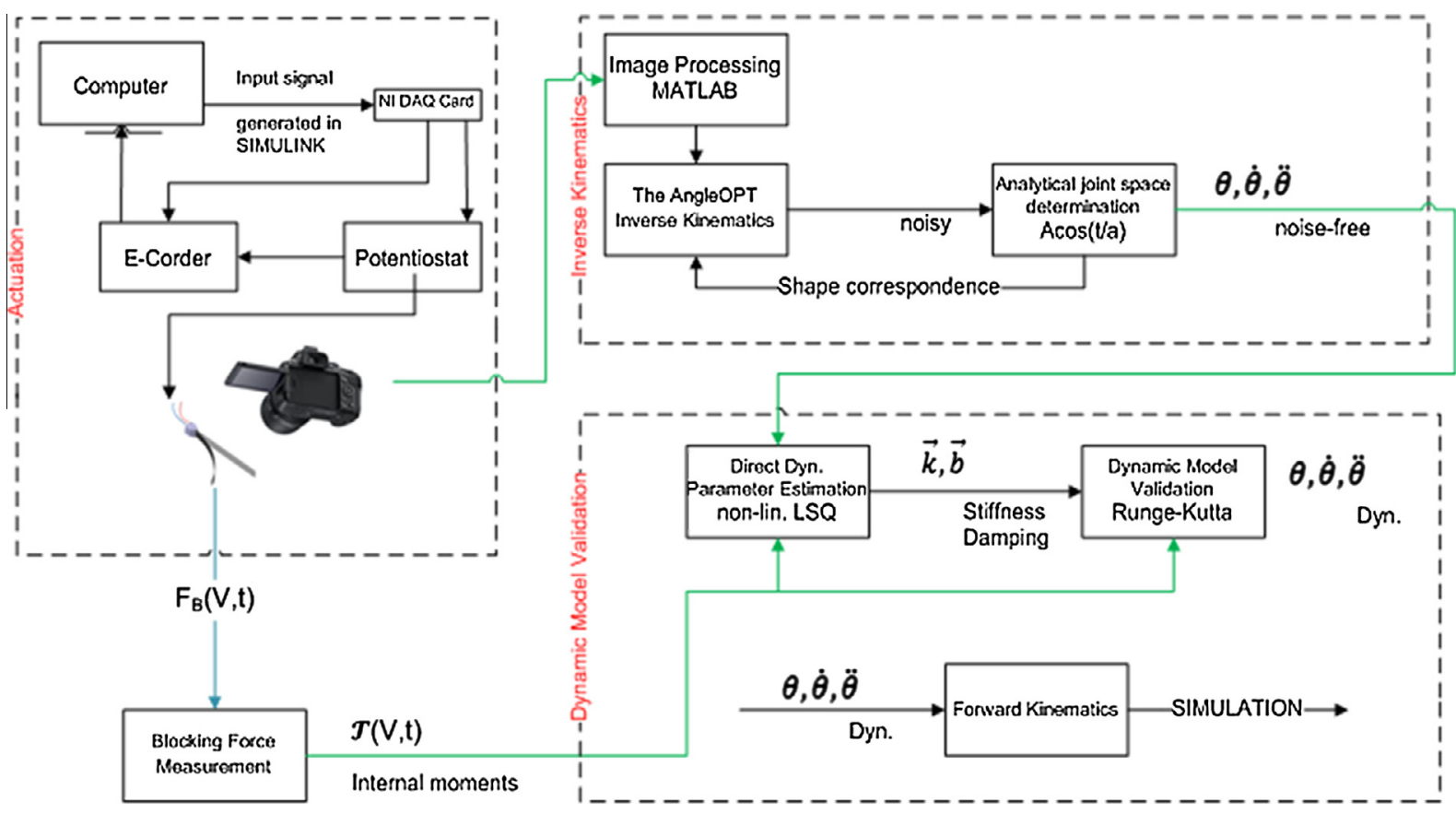

Fig. 4. Schematic of the experimental set-up and the electro-mechanical model validation. 

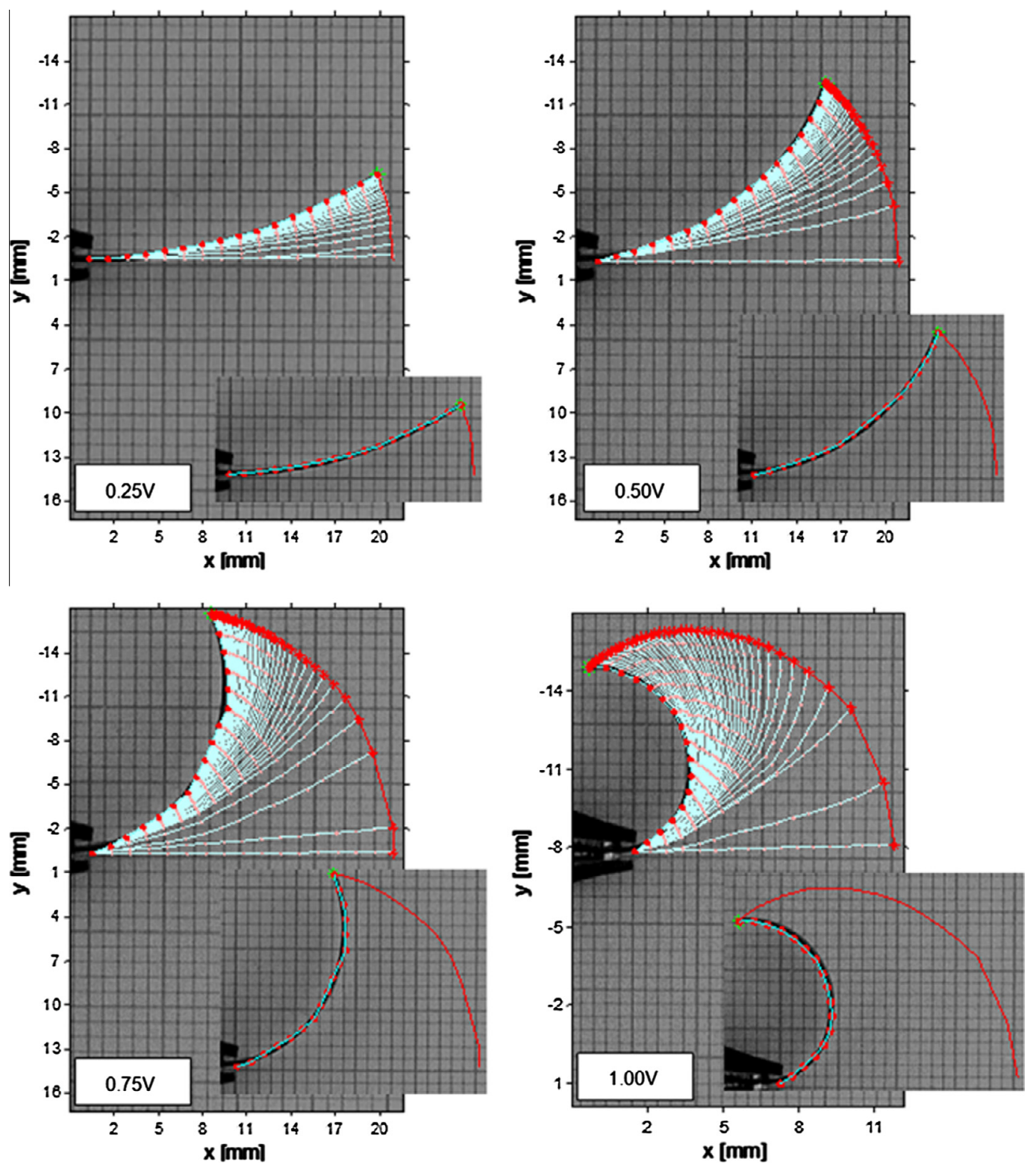

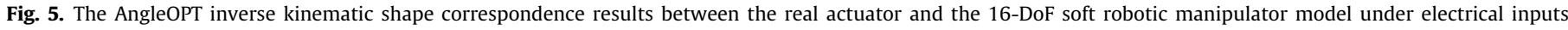
$0.0-1.0 \mathrm{~V}$.

adapted from soft robotic manipulator modelling) consisting of 16 serially connected rigid links with stiffness and damping parameters representing the visco-elasticity of the actuator. It must be noted that the number of degrees of freedom is chosen as 16 for the sake of convenience. Any appropriate high link number can be chosen to model the EAP actuator, however, the computation power required for calculating inverse kinematic shape estimations must be considered while choosing the number of degrees of freedom. After validating the inverse kinematic model solved by the AngleOPT which generates excellent shape correspondence results between the deflections of the real actuator and the 16-DoF soft robotic kinematic model, the dynamic model is built with the joint stiffness and damping parameters. The actuators used in this study were stimulated by electrical inputs ranging from $0.0 \mathrm{~V}$ to $1.0 \mathrm{~V}$.

\subsection{Experimental set-up}

The experimental set-up consists of actuation, blocking force measurement and image processing systems. The key elements of the experimental set-up and the electro-mechanical model validation algorithm are depicted in Fig. 4. The electrical input signals were generated using a SIMULINK program and passed through a USBtype NI-DAQ card (NI USB-6251) to a potentiostat. The electrical inputs were applied to the PPy-EAP actuator using a gold-coated clamp. This was used for two main reasons: (i) gold coating reduces corrosion and electrical resistance on the electrical contacts which are in contact with the electrolyte solution when they are placed on the EAP actuator and (ii) clamping the EAP actuator increases the contact area of the EAP actuator, which enhances the actuation capability. The motion of the PPy-EAP actuators was recorded using a digital camera (Nikon D5100). The videos were recorded at $30 \mathrm{fps}$, which was sufficient to record the bending motion of the actuators. The actuators were stimulated long enough to cover the total bending range of the PPy-EAP actuator; from its neutral (the actuator is straight and not activated) to its fully charged (maximum bent) state. After recording the actuator's bending motion under different electrical inputs, the tip displacement data was obtained by analysing videos using an image processing algorithm. 


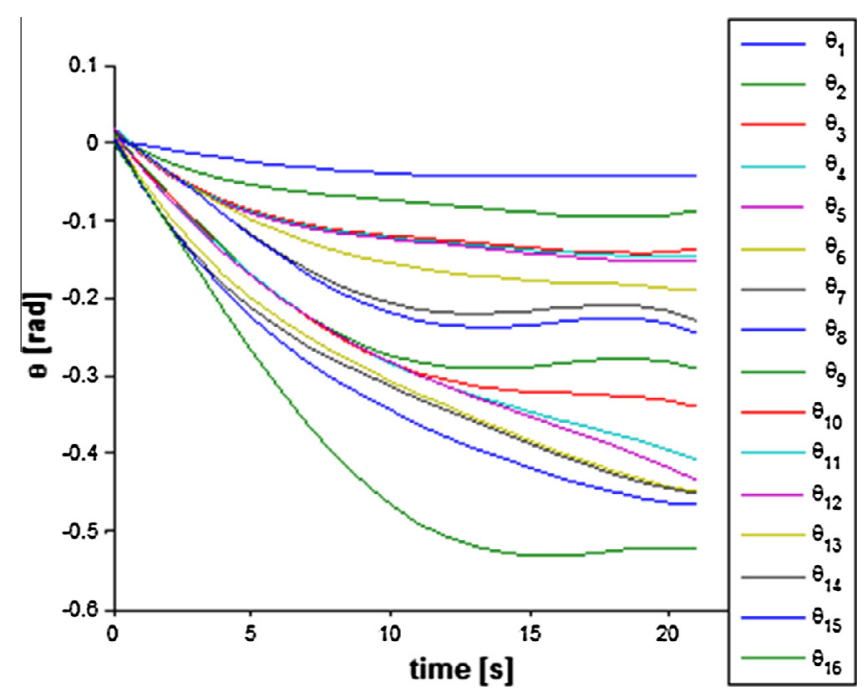

Fig. 6. The AngleOPT joint space inverse kinematic results under $0.25 \mathrm{~V}$ input voltage.

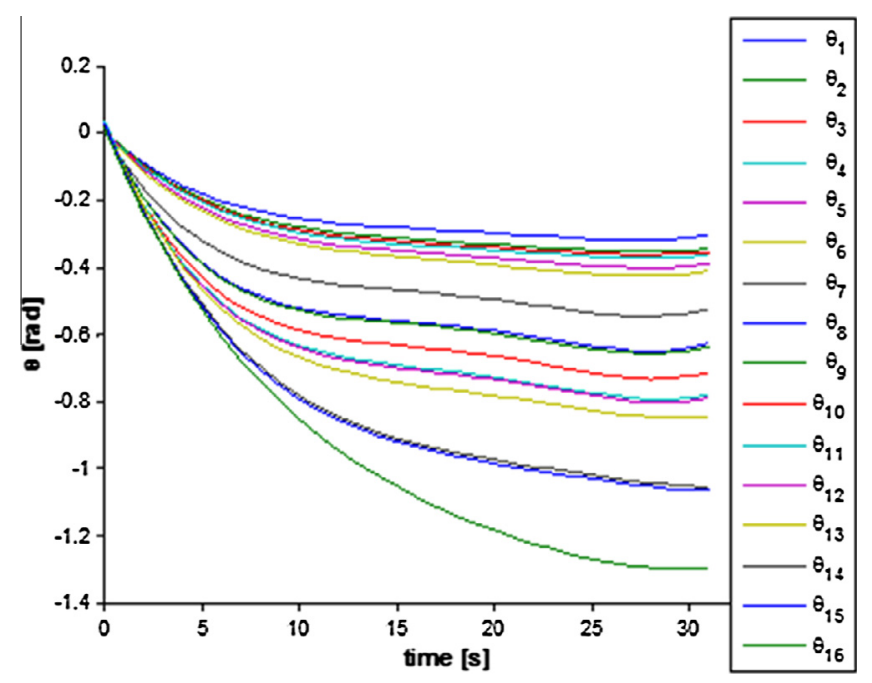

Fig. 7. The same as Fig. 6 , but for $0.50 \mathrm{~V}$ input voltage.

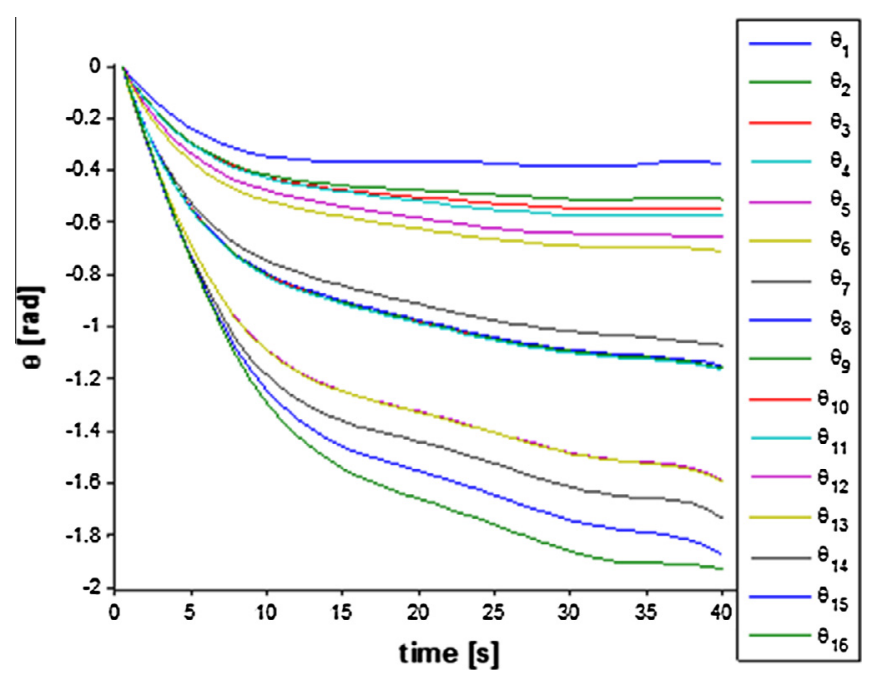

Fig. 8. The same as Fig. 6, but for $0.75 \mathrm{~V}$ input voltage.

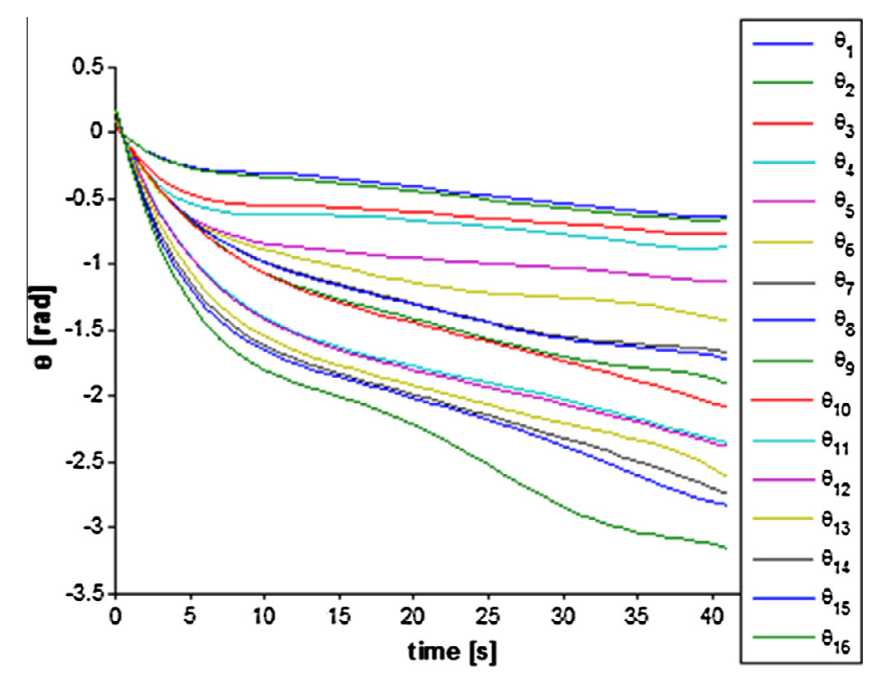

Fig. 9. The same as Fig. 6, but for $1.0 \mathrm{~V}$ input voltage.
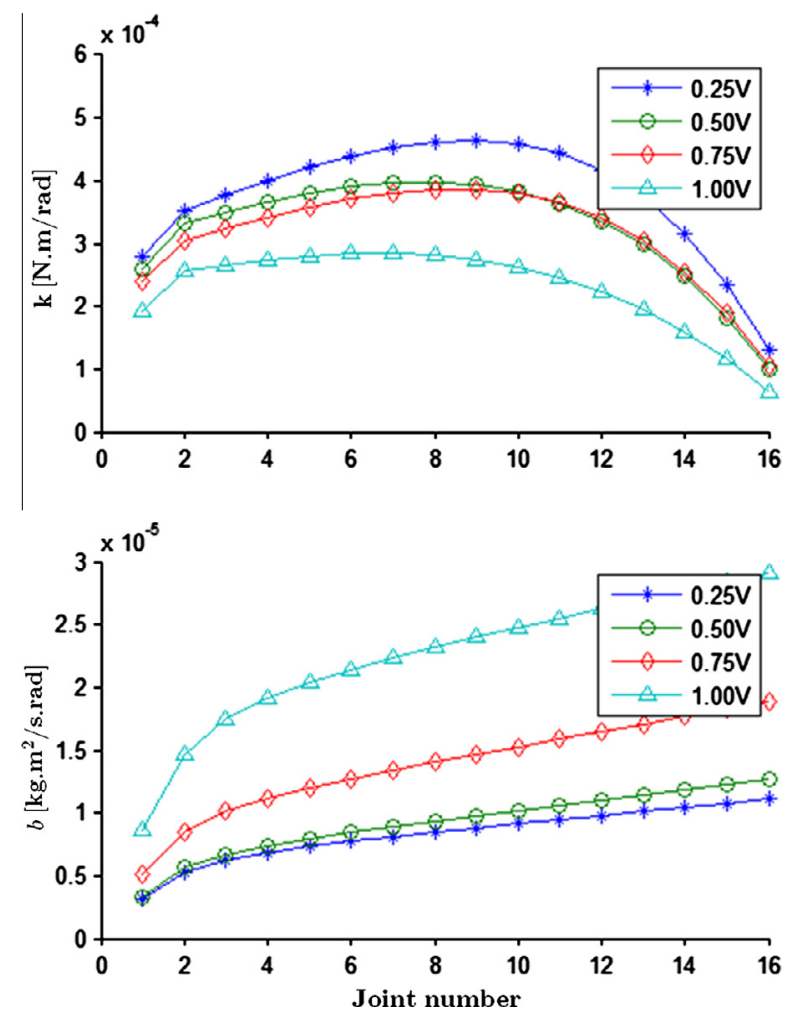

Fig. 10. Joint stiffness and damping values under $0.25-1.00 \mathrm{~V}$.

5.2. 16-DoF dynamic model for the soft robotic manipulator's electromechanical model

The electro-mechanical model (dynamic model and electrical relation to internal bending moments) considers the PPy-EAP actuator as a soft robotic manipulator with 16 rigid links serially connected by flexible and damped joints. This 16-DoF model is employed to identify joint stiffness and damping parameters using the experimental data obtained from the image processing system, data which is at the tip position of the PPy-EAP actuator. These tip positions were used as the inputs to the inverse kinematic model with a gradient-based self-built-constraint approach in order to determine the joint positions of the 16-DoF kinematic model. The 


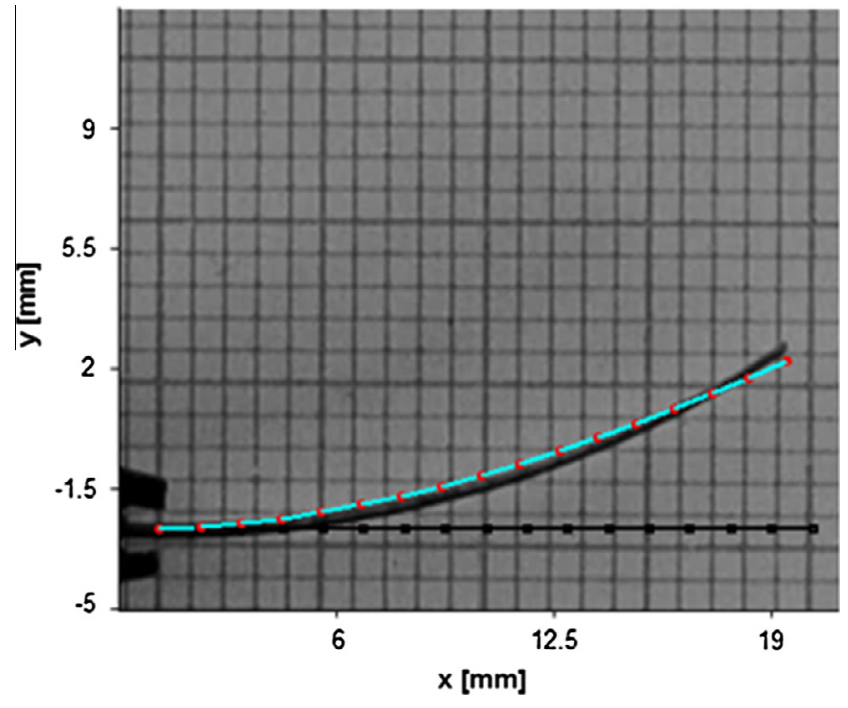

Fig. 11. Comparison between the 16-DoF electro-mechanical model (red dotted cyan curve) and the PPy-EAP actuator (thick black curve in the background image) under $0.25 \mathrm{~V}$ input voltage (black dotted line is the initial state of both the model and the actuator. (For interpretation of the references to colour in this figure legend, the reader is referred to the web version of this article.)

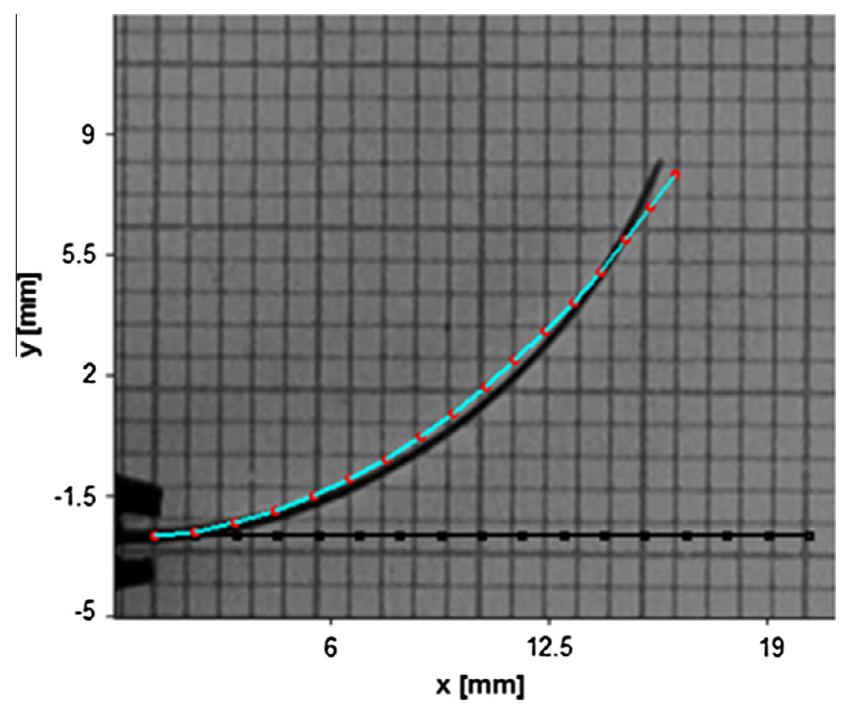

Fig. 12. The same as Fig. 11 , but for $0.50 \mathrm{~V}$ input voltage.

gradient-based self-built-constraint approach guides the AngleOPT inverse kinematic shape estimation to find the exact kinematic configuration of the PPy-EAP actuator by applying physical constraints continuously. These joint positions were used to determine the smooth joint trajectories of the actuator using Eqs. (19)-(21). The analytical joint space method was used for a number of reasons. First, it is an analytical method by which the joint angular velocity and angular accelerations can be directly determined from the mathematical expressions, Eqs. (19)-(21). Second, this method generates the joint trajectories smoothly and this helps estimate the joint stiffness and damping parameters more precisely using the direct dynamic parameter identification method. The inverse kinematic results corresponding to the joint trajectories of the actuator modelled as a $16-$ DoF soft robotic manipulator obtained from the AngleOPT algorithm are shown in Figs. 5-9 for electrical inputs from $0.0 \mathrm{~V}$ to $1.0 \mathrm{~V}$.

Next, these joint space trajectories are used as the reference trajectories in order to obtain the smoother joint trajectories

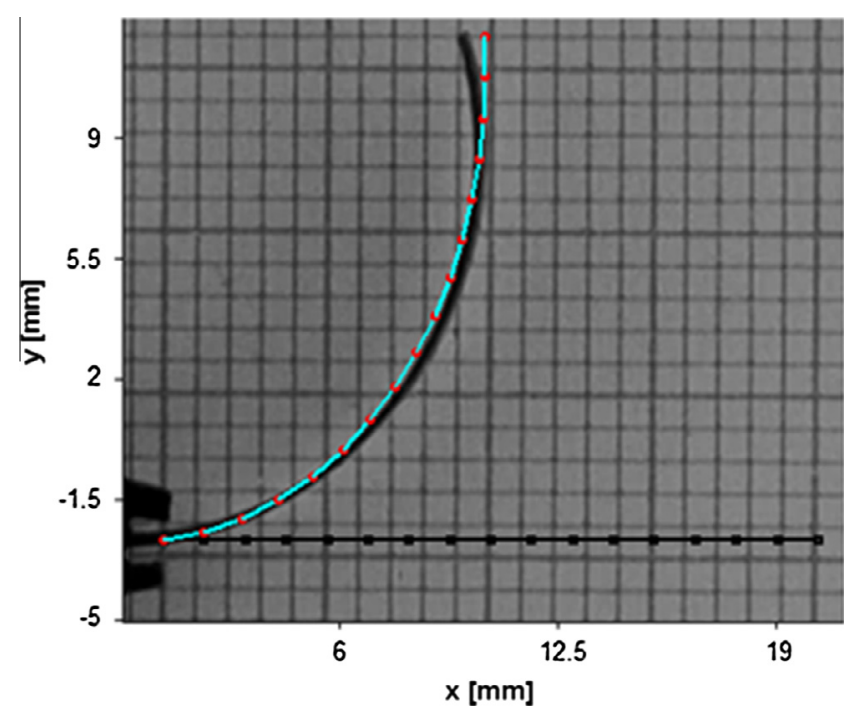

Fig. 13. The same as Fig. 11, but for $0.75 \mathrm{~V}$ input voltage.

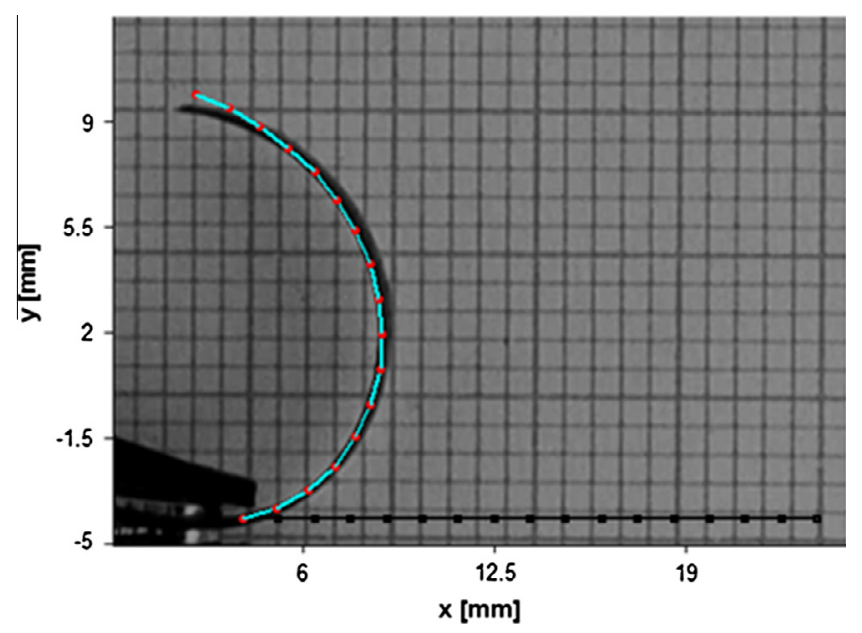

Fig. 14. The same as Fig. 11, but for $1.0 \mathrm{~V}$ input voltage.

analytically by employing Eqs. (19)-(21). The direct dynamic parameter identification method is then employed to estimate the joint stiffness and damping parameters of the PPy-EAP actuator's 16-DoF dynamic model. The joint stiffness and damping parameters are shown in Fig. 10 for the PPy-EAP actuator under electrical inputs: $0.25-1.0 \mathrm{~V}$ with a $0.25 \mathrm{~V}$ incremental input step at each test. It must be noted that the joint stiffness and damping parameters change with the input voltage. Calculating the stiffness or modulus of the elasticity of smart actuators is a challenging task. With the proposed modelling and parameter estimation method, this can be done effectively.

After identifying the joint stiffness and damping parameters of the PPy-EAP actuator, the electro-mechanical model is further validated using these joint stiffness values and damping parameters. The equations of motion for the actuator are solved numerically and the results are shown in Figs. 11-18, together with the real actuator configurations. To demonstrate the efficacy of the model in estimating the tip deflections, a new set of experimental results were generated to compare them with the numerical results obtained from the solution of the dynamic model with the identified parameters. The steady-state positions (final) of the actuator under $0.25 \mathrm{~V}, 0.50 \mathrm{~V}, 0.75 \mathrm{~V}$ and $1.00 \mathrm{~V}$ are shown in Figs. 11-14, which show an excellent match between the numerical and experimental results. 


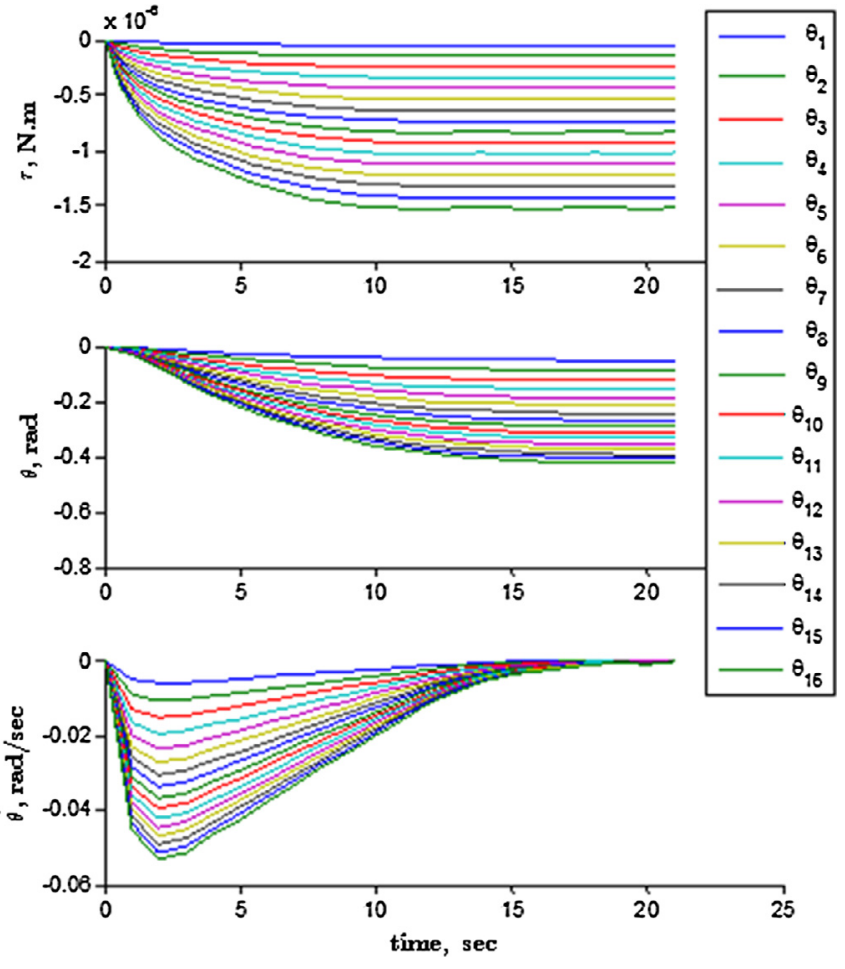

Fig. 15. The internal moment inputs, position and velocity results estimated from the electro-mechanical model of the PPy-EAP actuator under $0.25 \mathrm{~V}$ input voltage.

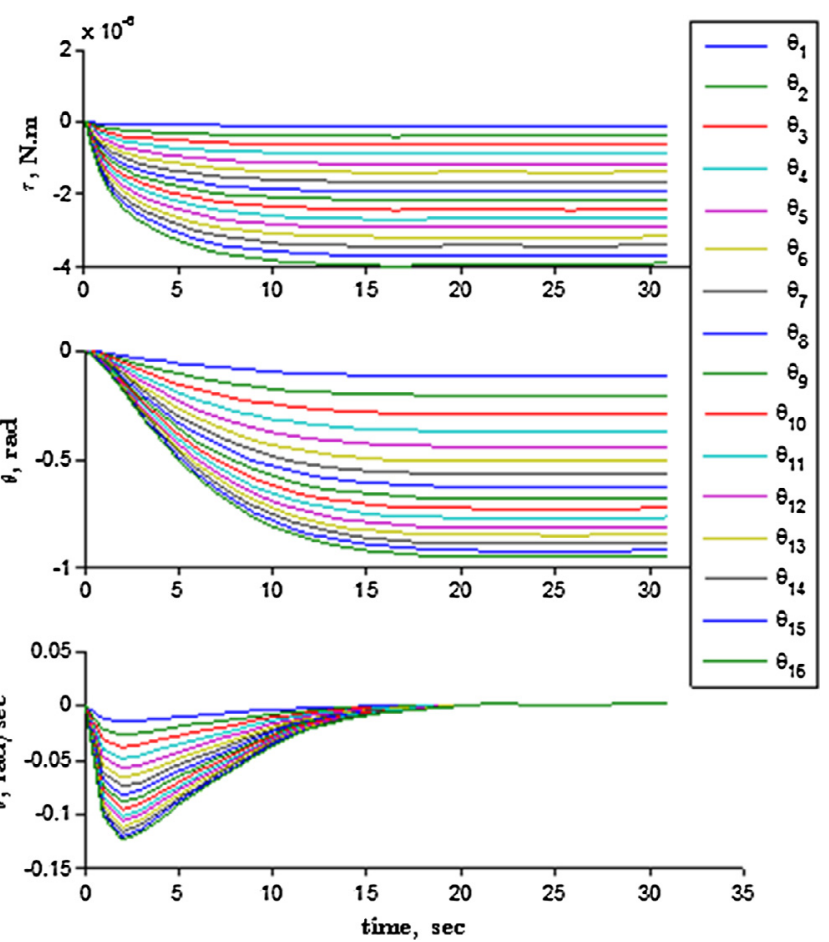

Fig. 16. The same as Fig. 15, but for $0.50 \mathrm{~V}$ input voltage.

\section{Discussion}

The proposed methodology employs soft robotic electromechanical modelling in order to estimate the highly non-linear bending behaviour of EAP actuators. The numerical solutions from the model show an excellent correlation with the experimental
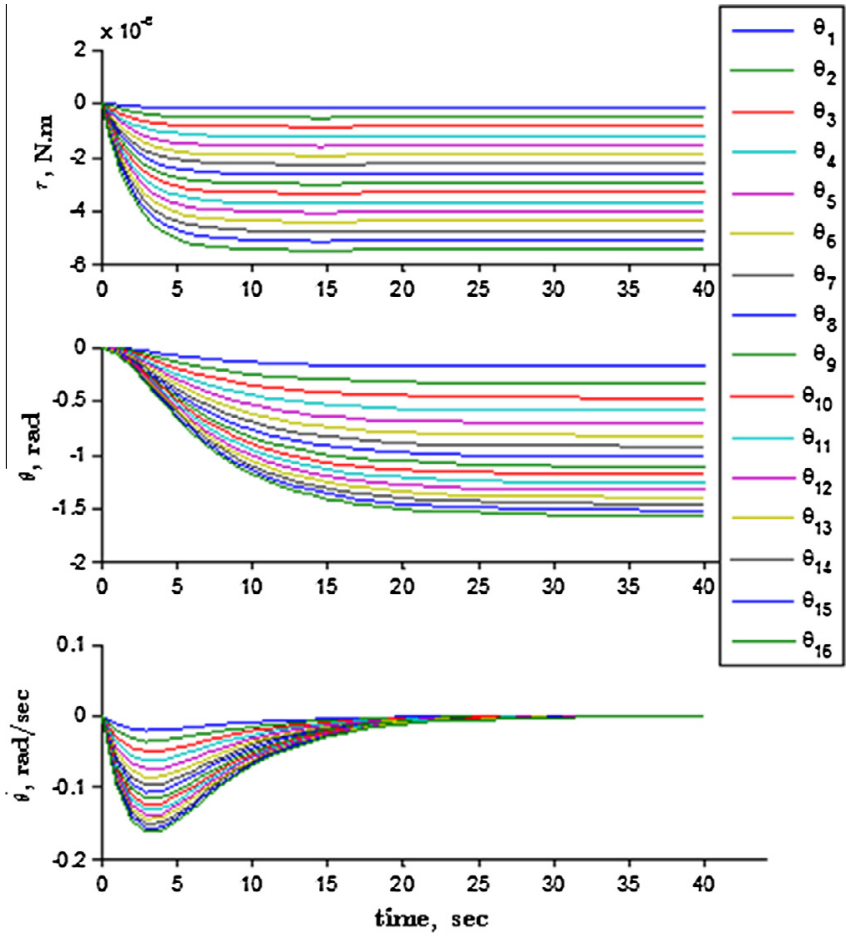

Fig. 17. The same as Fig. 15 , but for $0.75 \mathrm{~V}$ input voltage.
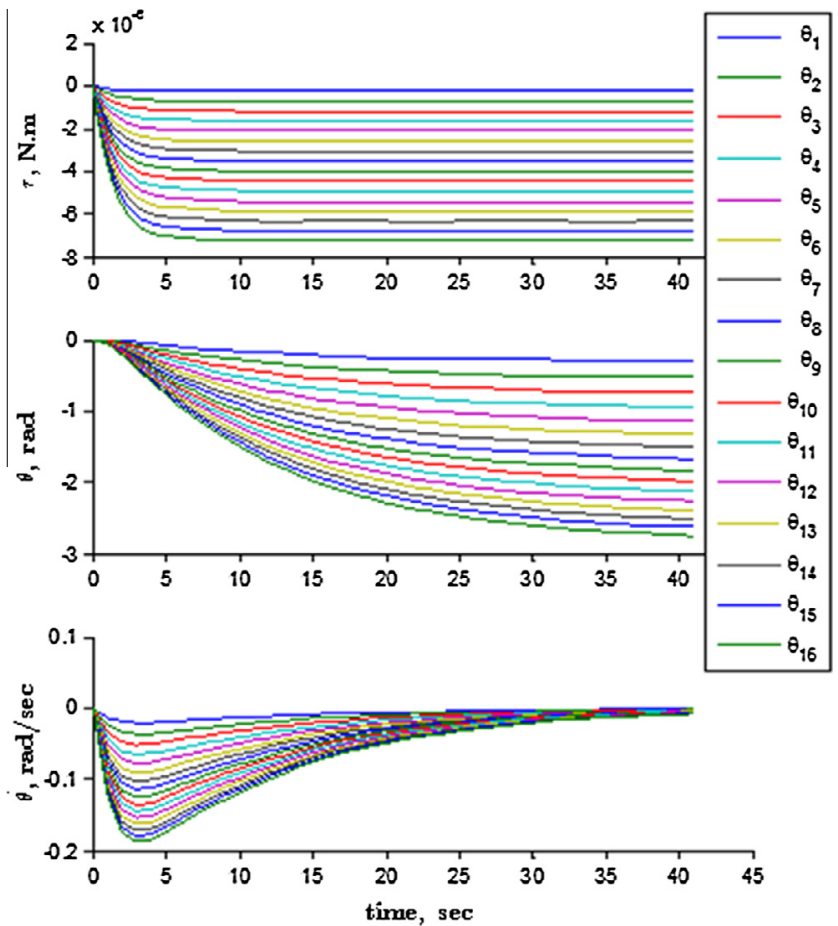

Fig. 18. The same as Fig. 15, but for $1.0 \mathrm{~V}$ input voltage.

results. Results suggest that this methodology is quite effective in identifying the dynamic parameters of the EAP actuator's electromechanical model, as well as in estimating the EAP actuator's whole shape variations dynamically under various electrical stimuli. This methodology employs an image processing system in order to obtain tip position data from the EAP actuator which is used in the inverse kinematic shape estimation process of the EAP actuator. The image processing system used in this study does 
not extract any data regarding the rest of the EAP actuator but only tip position data. Therefore, quantification of estimated results can be performed for tip position error and the quantification of inverse kinematic shapes of the EAP actuator estimated will be a topic of our future research by extending the image processing system in order to obtain the EAP actuator's whole shape data experimentally. Following that the extracted shape data of the EAP actuator will be used to determine imaginary joint positions in the kinematic model. It must be noted that an image is composed of scattered data which cannot be used directly to determine the imaginary joints in the kinematic model. However, these scattered data can be used to obtain a curve representing the configuration of the EAP actuator. This method might not require additional experimental arrangement, but further image analysis of the EAP actuator's motion. Alternatively, an indicator mark (e.g. red colour) can be used to locate the EAP actuator's imaginary joints by placing a coloured mark on the edge of the EAP actuator. Different colours can easily be identified from an image, without requiring further calculations in order to obtain the joint positions from the scattered image data. Even though this method will require some additional experimental arrangement, the extracted joint data can be used directly to quantify the inverse kinematic shape estimations (kinematic states) of the EAP actuator.

The proposed electro-mechanical modelling methodology is sensitive to experimental data measurements as it uses numerical optimization techniques to estimate the joint space positions and dynamic parameters of the PPy-EAP actuator: the smoother the experimental data is, the more accurately joint space and dynamic parameters can be estimated. Smoothing the joint space data with analytical functions, Eqs. (19)-(21), improves the accuracy of the dynamic parameter identification method. Although the results presented in this study were generated to step voltage inputs ranging from 0.0 to $1.0 \mathrm{~V}$, this can easily be extended to other types of inputs. The purpose of using step inputs is to analyse the PPy-EAP actuator's bending capabilities so that enough time and electrical stimulus is given to the actuator in order to let the actuator bend to its maximum bending position. The proposed electro-mechanical model uses the inverse kinematic solutions of the EAP actuator as inputs to calculate the visco-elastic properties of the actuator. Ideally, it would be preferable to calculate the kinematic and dynamic parameters of the actuator for a given input voltage. The input voltage can be included in the EAP actuator's dynamic model as the tip displacement is a function of time and input voltage by following the method described in our previous study [17].

\section{Conclusion and future work}

We have presented an effective methodology to analyse and model a PPy based EAP actuator's dynamic behaviour as a soft robotic manipulator with built-in actuation. This methodology consists of the backbone curve approach in soft robotic manipulators and its dynamic modelling, direct dynamic parameter estimation, and image processing algorithms. The effectiveness of this methodology has been demonstrated for tri-layer laminated PPyEAP actuators considered as a soft robotic actuator. The proposed method provides not only a reliable tool for estimating the actuator parameters but also solves the EAP actuator's inverse kinematics and dynamics problems in order to find their configurations as they vary over time. The electro-mechanical model presented in this paper improves our understanding of the highly non-linear bending behaviour of EAP actuators under higher electrical inputs (up to $\sim 1.0 \mathrm{~V}$ ). The PPy-EAP actuator's condition-dependent dynamic parameters have also been identified within the electromechanical model and experimental data. Further, the electro-mechanical model estimates the EAP actuator's whole bending behaviour both kinematically and dynamically. This has the potential to be of use in a broad range of applications, including active compliant mechanisms, biomedical applications and bio-inspired micro-robotic devices.

Future work includes validating the proposed modelling and identification methodology for actuators with different geometric parameters (length, width and thickness) and how the actuator parameters, including the modulus of elasticity, change with the geometric parameters. We also plan to evaluate the model for an EAP actuator manipulating a tip load. This methodology will be extended to modelling the dynamic behaviour of the EAP actuators forming 3D configurations so that they generate a configuration emerging out of the fabrication plane -from a $2 \mathrm{D}$ configuration to a 3D configuration.

\section{References}

[1] Alici G, Huynh NN. Performance quantification of conducting polymer actuators for real applications: a microgripping system. Mechatronics, IEEE/ ASME Trans 2007; 12:73-84.

[2] Alici G, Devaud V, Renaud P, Spinks G. Conducting polymer microactuators operating in air. J Micromech Microeng 2009;19:025017.

[3] Wu Y, Alici G, Spinks GM, Wallace GG. Fast trilayer polypyrrole bending actuators for high speed applications. Synth Met 2006;156:1017-22.

[4] Alici G, Spinks G, Huynh NN, Sarmadi L, Minato R. Establishment of a biomimetic device based on tri-layer polymer actuators-propulsion fins. Bioinspiration Biomimetics 2007;2:18-30.

[5] McGovern S, Alici G, Truong V-T, Spinks G. Finding NEMO (novel electromaterial muscle oscillator): a polypyrrole powered robotic fish with real-time wireless speed and directional control. Smart Mater Struct 2009;18:095009.

[6] Alici G, Gunderson D. A bio-inspired robotic locomotion system based on conducting polymer actuators. In: Advanced Intelligent Mechatronics, 2009. AIM 2009. IEEE/ASME International Conference on; 2009. p. 998-1004.

[7] Mutlu R, Alici G. Artificial muscles with adjustable stiffness. Smart Mater Struct 2010;19:045004.

[8] Mutlu R, Alici G. A multistable linear actuation mechanism based on artificial muscles. J Mech Des 2010;132:111001.

[9] Alici G, Spinks GM, Madden JD, Yanzhe W, Wallace GG. Response characterization of electroactive polymers as mechanical sensors. Mechatronics, IEEE/ASME Trans 2008;13:187-96.

[10] Pei Q Inganaes O. Electrochemical applications of the bending beam method. 1. mass transport and volume changes in polypyrrole during redox. J Phys Chem 1992;96:10507-14. 1992/12/01.

[11] Benslimane M, Gravesen P, West K, Skaarup S, Sommer-Larsen P. Performance of polymer-based actuators: the three-layer model. In: Bar-Cohen Y, editor. Proc. SPIE, Smart structures and materials 1999: electroactive polymer actuators and devices, vol. 3669, 1999. p. 87-97.

[12] Madden PGA. Development and modeling of conducting polymer actuators and the fabrication of a conducting polymer based feedback loop. Ph.D. thesis, Dept. of Mechanical Engineering, Massachusetts Institute of Technology; 2003.

[13] Alici G, Mui B, Cook C. Bending modeling and its experimental verification for conducting polymer actuators dedicated to manipulation applications. Sens Actuators A: Phys 2006;126:396-404.

[14] Alici G. An effective modelling approach to estimate nonlinear bending behaviour of cantilever type conducting polymer actuators. Sens Actuators B: Chem 2009;141:284-92.

[15] Dorf RC, Bishop RH. Mathematical models of systems. In: Modern control systems. NJ: Pearson Prentice Hall; 2008. p. 41-143.

[16] Mutlu R, Alici G, Weihua L. Kinematic analysis of electroactive polymer actuators as soft and smart structures with more DoF than inputs. In: Advanced Intelligent Mechatronics (AIM), 2012 IEEE/ASME International Conference on; 2012. p. 484-9.

[17] Mutlu R, Alici G, Li W. An effective methodology to solve inverse kinematics of electroactive polymer actuators modelled as active and soft robotic structures. Mech Machine Theory 2013;67:94-110.

[18] Bar-Cohen Y. Electroactive polymer (EAP) actuators as artificial muscles: reality, potential, and challenges. 2nd ed. Bellingham: SPIE Press; 2004.

[19] Smela E. Conjugated polymer actuators for biomedical applications. Adv Mater 2003;15:481-94.

[20] Mutlu R, Alici G, Weihua L. Kinematic modeling for artificial flagellum of a robotic bacterium based on electroactive polymer actuators. In: Advanced Intelligent Mechatronics (AIM), 2011 IEEE/ASME International Conference on; 2011. p. 440-5.

[21] Hirose S, Mori M. Biologically Inspired Snake-like Robots. In: Robotics and Biomimetics, 2004. ROBIO 2004. IEEE International Conference on; 2004. p. 17.

[22] Yekutieli Y, Sagiv-Zohar R, Aharonov R, Engel Y, Hochner B, Flash T. Dynamic model of the octopus arm. I. biomechanics of the octopus reaching movement. J Neurophysiol 2005;94:1443-58. 
[23] Hannan MW, Walker ID. Novel kinematics for continuum robots. In: Lenarčič ] Stanišić MM, editors. Advances in robot kinematics. Netherlands: Springer; 2000. p. 227-38.

[24] Hannan MW, Walker ID. Kinematics and the Implementation of an Elephant's trunk manipulator and other continuum style robots. J Robotic Syst 2003;20:45-63.

[25] Mochiyama H, Shimemura E, Kobayashi H. Shape correspondence between a spatial curve and a manipulator with hyper degrees of freedom. In: Intelligen Robots and Systems, 1998. Proceedings., 1998 IEEE/RSJ International Conference on; 1998. p. 161-6.

[26] Chirikjian GS. A continuum approach to hyper-redundant manipulator dynamics. In: Intelligent Robots and Systems '93, IROS '93. Proceedings of the 1993 IEEE/RSJ International Conference on; 1993. p. 1059-66.

[27] Chirikjian GS. Inverse kinematics of binary manipulators using a continuum model. J Intell Robotics Syst 1997;19:5-22.

[28] Toponogov VA. Theory of curves in three-dimensional euclidean space and in the plane. In: Rovenski V, editor. Differential geometry of curves and surfaces. Boston: Birkhäuser; 2006. p. 1-63.
[29] Tokhi O, Azad AKM. Dynamic characterisation of flexible manipulators using symbolic manipulation. In: Flexible robot manipulators: modelling, simulation and control. Hertfordshire (UK): Institution of Engineering and Technology; 2008. p. 119-46.

[30] Shabana AA. Analytical techniques. In: Dynamics of multibody systems. NY: Cambridge University Press; 2005. p. 85-158.

[31] Madden JD, Cush RA, Kanigan TS, Hunter IW. Fast contracting polypyrrole actuators. Synth Met 2000;113:185-92.

[32] Yu Y-Q, Feng Z-L, Xu Q-P. A pseudo-rigid-body 2R model of flexural beam in compliant mechanisms. Mech Machine Theory 2012; 55:18-33.

[33] Kozłowski K. Experimental identification of robot dynamic parameters. In: Modelling and identification in robotics. NY: Springer-Verlag; 1998. p. $131-88$.

[34] Depince P. Parameters identification of flexible robots. In: Robotics and Automation, 1998. Proceedings. 1998 IEEE International Conference on, 1998. p. $1116-21$. 